\title{
The Use of Once-Daily Dosing of Gentamicin in Obstetrics and Gynecology
}

\author{
H.C. Wiesenfeld* and R.P. Heine \\ Department of Obstetrics, Gynecology, and Reproductive Sciences, University of Pittsburgh School of \\ Medicine/Magee-Womens Research Institute, Pittsburgh, PA
}

\begin{abstract}
Gentamicin is a widely-used antimicrobial agent for obstetric and gynecologic infections. Renewed excitement in this antibiotic has arisen from recent information supporting less frequent dosing. In this symposium, we will describe the pharmacokinetics of gentamicin and review new information advocating the use of once-daily administration of gentamicin. Infect. Dis. Obstet. Gynecol. 6:155159, 1998. @ 1998 Wiley-Liss, Inc.
\end{abstract}

KEY WORDS

gentamicin; antimicrobials; gynecologic infection

A minoglycosides, discovered over half a century ago, remain among the most frequently prescribed antibiotic agents for serious infections. Aminoglycosides are an important component of the obstetrician-gynecologist's armamentarium for obstetrical, gynecological, and postoperative infections. Gentamicin is the most widely used aminoglycoside in these settings, and thus will be the focus of this paper. While aminoglycosides have been in use for several decades, recent information supporting the administration of these agents once a day has brought renewed interest in this "older" class of compounds for this modern, cost-conscience medical era.

\section{STRUCTURE AND DERIVATION}

Gentamicin is a fermentation product derived from the soil actinomycetes Micromonospora purpurea and Micromonospora echinospora. All aminoglycosides are comprised of an essential six-membered ring with amino-group constituents bound to at least two sugar moieties. Gentamicin is soluble in water but insoluble in organic solvents, thereby re- stricting permeability across the lipid-rich cell membranes.

\section{MECHANISM OF ACTION}

Antimicrobial activity is achieved following a multistep process that involves binding to the cell surface, uptake into the intracellular compartment, and, finally, binding and interaction with ribosomes. Gentamicin binds passively to lipopolysaccharides, phospholipids, and other protein constituents of the outer membrane of gram-negative bacteria. This binding causes disruption of the outer membrane, resulting in increased permeability of the cell wall. Uptake of gentamicin subsequently occurs by energy-dependent processes. Gentamicin remains intracellular, with intracellular concentrations far greater than those found in the extracellular space. The final step culminating in antimicrobial activity occurs with binding of gentamicin to the bacterial ribosome (between the smaller [30S] and larger [50S] subunits), resulting in misreading of messenger RNA and impaired protein synthesis.

*Correspondence to: Dr. Harold C. Wiesenfeld, Department of Obstetrics, Gynecology, and Reproductive Sciences, MageeWomens Hospital, 300 Halket Street, Pittsburgh, PA 15213. E-mail: hwiesenfeld@mail.magee.edu 


\section{PHARMACOKINETICS}

Gentamicin, as all aminoglycosides, is administered by the parenteral route (intravenously or intramuscularly) and is poorly absorbed when taken by mouth. Its high water solubility enables wide distribution in the vascular and interstitial spaces. The volume of distribution $\left(\mathrm{V}_{\mathrm{D}}\right)$ is $0.2-0.3 \mathrm{~L} / \mathrm{kg}$ but may be greater in patients with severe infections. The half-life $\left(\mathrm{T}_{1 / 2}\right)$ of gentamicin is $2 \pm 1$ hours. Elimination occurs almost entirely by the kidney, where it is excreted unchanged with minimal reabsorption. Urinary concentration typically exceeds peak plasma levels during administration, and urinary levels following a single dose surpass therapeutic levels for several days.

The pharmacokinetics of gentamicin involve three phases. Following parenteral administration, the drug is distributed from the intravascular to the extravascular space over 15 to 30 minutes. During the second phase, gentamicin is excreted into the kidneys, a process related to glomerular filtration rate, with the resultant half-life closely dependent on renal function. The final phase involves the excretion of gentamicin from renal tissue.

Gentamicin is widely used in obstetrics and gynecology, as gram-negative organisms are frequently implicated in reproductive tract infections, either alone or as part of a polymicrobial process. Gentamicin is often selected to treat chorioamnionitis, postpartum endometritis, pyelonephritis, pelvic inflammatory disease, and tuboovarian abscesses. Among obstetric patients there are wide variations in gentamicin elimination, posing difficulties in dose calculation. Using a dose of $1 \mathrm{mg} / \mathrm{kg}$ every 8 hours, Duff et al. found that one-third of women with postpartum endometritis had subtherapeutic levels of gentamicin. ${ }^{1}$ Zaske et al. estimated that $49 \%$ of patients receiving standard doses of gentamicin would have subtherapeutic levels. ${ }^{2}$ Establishing therapeutic levels required a wide range of doses of gentamicin $(3.0$ to $11.6 \mathrm{mg}$ / $\mathrm{kg} / \mathrm{d}$ ). Given the wide variation of doses among obstetric patients, when multiple daily dosing of gentamicin is used, serum concentrations should be monitored in patients who do not quickly respond to therapy.

\section{SIDE EFFECTS}

The clinician's main concern regarding the use of gentamicin (and other aminoglycosides) is the risk of toxicity, specifically nephrotoxicity and ototoxicity. The incidence of nephrotoxicity is between 5 and $10 \%$ and is even less frequently seen in otherwise healthy individuals receiving gentamicin. Risk factors for nephrotoxicity include older age, multisystem disease, preexisting renal disease, concomitant administration of nephrotoxic agents (eg, cis-platinum, amphotericin-B), and multiple daily dosing (see below). Aminoglycoside-related nephrotoxicity rarely results in severe renal compromise requiring dialysis. Gentamicin should be discontinued if nephrotoxicity occurs; resolution of renal function will subsequently follow over several days. When it is ill-advised to stop aminoglycoside administration, the dosage should be adjusted to reduce the risk of increasing toxicity.

\section{SPECTRUM OF ACTIVITY}

Gentamicin is active against the majority of gramnegative bacteria, such as Escherichia coli, Klebsiella sp, Proteus mirabilis, Citrobacter sp, Serratia sp, Enterobacter sp, Hemophilus influensae, and some strains of Pseudomonas aeruginosa. It possesses some activity against gram-positive organisms, particularly methicillin-susceptible Staphylococcus aureus. Gentamicin is not active against anaerobic organisms.

\section{RESISTANCE}

Resistance against aminoglycosides occurs by several mechanisms, including enzymatic alteration of the aminoglycoside by aerobic gram-negative bacteria, altered bacterial uptake, and by modification of ribosomal binding sites. Resistance of a particular organism to gentamicin is slow in development, therefore resistance rarely emerges during a therapeutic course of gentamicin. Resistance to gentamicin is observed in many strains of enterococci, Pseudomonas aeruginosa, and methicillin-resistant Staphylococcus aureus.

\section{ONCE-DAILY DOSING OF GENTAMICIN}

Recently developed broad-spectrum antimicrobial agents (eg, cephalosporins and fluoroquinolones) with less frequent dosing requirements and favorable safety profiles have supplanted aminoglycosides in many clinical settings. However, over the years new information on the pharmacokinetic properties and killing parameters of gentamicin have enabled the development of alternative, less frequent dosing regimens with lower risk of toxic- 
ity. Aminoglycosides possess concentration, or dose-dependent killing properties, with greater bacteriocidal activity associated with higher concentrations of drug (eg, 10 times the minimum inhibitory concentration [MIC]). This contrasts with $\beta$-lactam agents that possess time-dependent killing, where maximum antibacterial activity occurs during prolonged periods of drug concentration above the MIC. Additionally, gentamicin has a "postantibiotic effect" (PAE), which is defined as persistent bacterial growth inhibition after antibiotic exposure has ceased. In vitro and animal studies have documented equivalent or greater antimicrobial activity with once-daily dosing versus smaller, more-frequent doses of aminoglycosides. $^{3,4}$ The PAE of aminoglycosides pertains to several different microbial species, and the higher the initial aminoglycoside level, the greater the PAE. Both the PAE and the concentrationdependent killing properties of aminoglycosides are the rationale behind the utilization of oncedaily dosing of gentamicin. Specifically, the single large daily dose maximizes bacterial killing, while the PAE during the extended dosing interval, when the drug level in the serum is low, would either allow for ongoing killing or at least prevent regrowth of bacteria.

There has been a rapid accumulation of published trials of once-daily dosing of aminoglycosides, including netilmicin, amikacin, and gentamicin. A small randomized study comparing onceand thrice-daily dosing of gentamicin or netilmicin in sixty patients demonstrated similar efficacy, but the study was limited by small sample size. ${ }^{5}$ Prins et al. compared once-daily dosing of gentamicin, using a dose of $4 \mathrm{mg} / \mathrm{kg} / \mathrm{d}$, to thrice-daily doses of $1.33 \mathrm{mg} / \mathrm{kg}$ of gentamicin among 67 adult patients with serious infections. Once-daily dosing was as effective as thrice-daily doses, with favorable clinical outcomes seen in $91 \%$ of patients receiving once-daily dosing and in $78 \%$ of those receiving conventional dosing. ${ }^{6}$ Similar results have been obtained with netilmicin and other aminoglycosides. ${ }^{7}$ Metaanalyses have repeatedly demonstrated equal efficacy of once-daily dosing of aminoglycosides compared with multiple daily dosing in both clinical and microbiologic cure rates. ${ }^{8-12}$ Additional studies are needed among certain populations, particularly pregnant women, children, and patients with endocarditis.
Toxicity studies of once-daily dosing of aminoglycosides have not revealed an increased risk of either nephrotoxicity or ototoxicity. While aminoglycosides are accumulated in the renal cortex, the uptake is saturable, with greater accumulation occurring in continuous or divided-dose infusions than in one large dose. Less nephrotoxicity was observed in laboratory animals receiving oncedaily dosing. In a review of once-daily aminoglycoside administration to over 2,000 adult patients, nephrotoxicity was observed in $1.2 \%$ of patients, and only three patients exhibited clinical ototoxicity. ${ }^{13}$ Prins et al. noted significantly less nephrotoxicity in patients with serious infections receiving the once-daily dose of gentamicin compared with thrice-daily dosing (5\% vs. $24 \%, P=0.016)$ and no difference in ototoxicity as assessed clinically and by audiometry. ${ }^{6}$ Similarly, several metaanalyses of single daily dosing of aminoglycosides failed to demonstrate increased nephrotoxicity or ototoxicity. ${ }^{8,9,11,12}$ However, in patients with preexisting renal failure, there are limited data on the safety of once-daily dosing.

A consensus is lacking regarding the timing, and even utility, of monitoring aminoglycoside levels. After 48 hours of therapy, Prins et al. obtained trough levels immediately preceding and peak levels 30 minutes following the next dose. ${ }^{6}$ The dose was subsequently lowered when the trough levels were above $2 \mathrm{mg} / \mathrm{L}$; no adjustment was made for peak levels. In a large series of adult patients receiving once-daily aminoglycosides (mostly gentamicin), Nicolau et al. reported that only $5 \%$ of patients required a dose adjustment. ${ }^{13}$ Based on his results, the following patient criteria for withholding aminoglycoside levels were identified: age less than 60 years, no concurrent therapy with nephrotoxic agents, no exposure to contrast media, no requirement for intensive care support, and not quadriplegics nor amputees. Nonetheless, serum creatinine was monitored every 2 to 3 days.

Experience with single-dose gentamicin among general adult populations has been the foundation for its use for reproductive tract infections. In our report in this issue of the journal, we evaluated the pharmacokinetics of once-daily gentamicin in women with postpartum endometritis. ${ }^{14}$ Using a single daily dose of $4.5 \mathrm{mg} / \mathrm{kg}$ actual body weight, peak gentamicin levels were above $10 \mathrm{mg} / \mathrm{L}$, with consistently low trough levels. Clinical efficacy of 
this regimen in this preliminary study was excellent, with no nephrotoxicity or clinical ototoxicity. A recently published randomized trial of once-daily $(5 \mathrm{mg} / \mathrm{kg} / \mathrm{d})$ vs. 8 -hour dosing $(1.75 \mathrm{mg} / \mathrm{kg}$ every 8 hours) of gentamicin for the treatment of postpartum endometritis demonstrated equal efficacy and cost savings with the once-daily regimen. ${ }^{15}$ Mitra et al. reported on once-daily gentamicin with twicedaily clindamycin versus gentamicin and clindamycin each administered three times a day for puerperal endometritis. The less-frequent dosing schedule was associated with similar outcomes to those with conventional dosing. ${ }^{16}$ Given the difficulty in establishing therapeutic levels with multiple daily doses in obstetric patients, once-daily dosing of gentamicin is appealing in this population. There are no data on once-daily gentamicin for gynecologic infections, although this regimen is becoming widely used in many centers. Similarly, once-daily dosing has not been well studied in pregnant women. Its safety in breastfeeding women remains to be established, however infant absorption from breast milk should not be a concern due to the inability of gentamicin to be absorbed from the gastrointestinal tract. There are limited data on the utility of performing routine peak and trough levels. As noted above, levels are likely unwarranted in otherwise healthy adults receiving once-a-day gentamicin for limited periods of time.

\section{COST}

Once-daily dosing of gentamicin offers important cost savings over traditional dosing schedules. Reduced costs are realized by a reduction in labor costs, particularly in pharmacist time and nursing labor, and material costs (intravenous tubing and bags). In contrast to the thrice-daily regimen, single-daily dosing does not require monitoring serum levels of gentamicin, resulting in further cost savings. We calculated a $44 \%$ reduction in patient charges with once-daily dosing of gentamicin for 72 hours of therapy. ${ }^{14}$ Substantial cost savings were similarly noted in the studies by Del Priore et al. and Mitra et al. ${ }^{15,16}$

\section{SUMMARY}

Gentamicin remains a widely used antibiotic for treating obstetric and gynecologic infections because of its activity against gram-negative organ- isms. Among obstetric patients, serum levels are varied, requiring monitoring of serum gentamicin concentrations to ensure therapeutic levels. There is substantial evidence among general adult populations documenting equal efficacy and reduced toxicity with once-daily dosing compared to standard multidose regimens. Limited data support the use of single-daily dose gentamicin for the treatment of postpartum endometritis. Using this familiar antibiotic in a new way results in an equally effective, safer, and less costly approach to the treatment of gram-negative infections.

\section{REFERENCES}

1. Duff P, Jorgensen JH, Gibbs RS, Blanco JD, Alexander G, Castaneda YS: Serum gentamicin levels in patients with post-cesarean endomyometritis. Obstet Gynecol 61:723-727, 1983.

2. Zaske DE, Cipolle RJ, Strate RG, Malo JW, Koszalka MF Jr: Rapid gentamicin elimination in obstetric patients. Obstet Gynecol 56:559-564, 1980.

3. Blaser J, Stone BB, Zinner SH: Efficacy of intermittent versus continuous administration of netilmicin in a twocompartment in vitro model. Antimicrob Agents Chemother 27:343-349, 1985.

4. Herscovici L, Grise G, Thauvin G, Lemeland JF, Fillastre JP: Efficacy and safety of once daily versus intermittent dosing of tobramycin in rabbits with acute pyelonephritis. Scand J Infect Dis 20:205-212, 1988.

5. Nordstrom L, Ringberg H, Cronberg S, Tjernstrom O, Walder M: Does administration of an aminoglycoside in a single daily dose affect its efficacy and toxicity? J Antimicrob Chemother 25:159-173, 1990.

6. Prins JM, Buller HR, Kuijper EJ, Tange RA, Speelman $\mathrm{P}$ : Once versus thrice daily gentamicin in patients with serious infections. Lancet 341:335-339, 1993.

7. de Vries PJ, Verkooyen RP, Leguit P, Verbrugh HA: Prospective randomized study of once-daily versus thrice-daily netilmicin regimens in patients with intraabdominal infections. Eur J Clin Microbiol Infect Dis 9:161-168, 1990.

8. Ali MZ, Goetz MB: A meta-analysis of the relative efficacy and toxicity of single daily dosing versus multiple daily dosing of aminoglycosides. Clin Infect Dis 24:796809, 1997.

9. Bailey TC, Little JR, Littenberg B, Reichley RM, Dunagan WC: A meta-analysis of extended-interval dosing versus multiple daily dosing of aminoglycosides. Clin Infect Dis 24:786-795, 1997.

10. Barza M, Ioannidis JP, Cappelleri JC, Lau J: Single or multiple daily doses of aminoglycosides: a meta-analysis BMJ 312:338-345, 1996. 
11. Hatala R, Dinh T, Cook DJ: Once-daily aminoglycoside dosing in immunocompetent adults: a meta-analysis. Ann Intern Med 124:717-725, 1996.

12. Hatala R, Dinh TT, Cook DJ: Single daily dosing of aminoglycosides in immunocompromised adults: a systematic review. Clin Infect Dis 24:810-815, 1997.

13. Nicolau DP, Wu AH, Finocchiaro S, et al.: Once-daily aminoglycoside dosing: impact on requests and costs for therapeutic drug monitoring. Ther Drug Monitoring 18: 263-266, 1996.

14. Sunyecz JA, Wiesenfeld HC, Heine RP: The pharmacokinetics of once-daily dosing with gentamicin in women with postpartum endometritis. Infect Dis Obstet Gynecol 6:160-162, 1998.

15. Del Priore G, Jackson-Stone M, Shim EK, Garfinkel J, Eichmann MA, Frederiksen MC: A comparison of oncedaily and 8-hour gentamicin dosing in the treatment of postpartum endometritis. Obstet Gynecol 87:994-1000, 1996.

16. Mitra AG, Whitten AG, Laurent SL, Anderson WE: A randomized. prospective study comparing once-daily gentamicin versus thrice-daily gentamicin in the treatment of puerperal infection. Am J Obstet Gynecol 177: 786-791, 1997. 


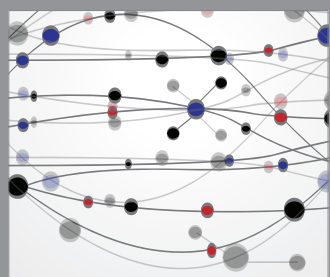

The Scientific World Journal
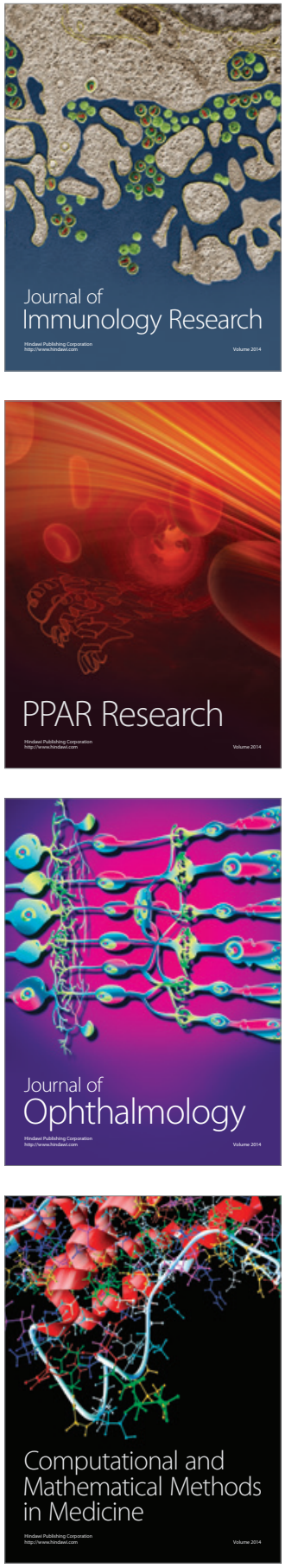

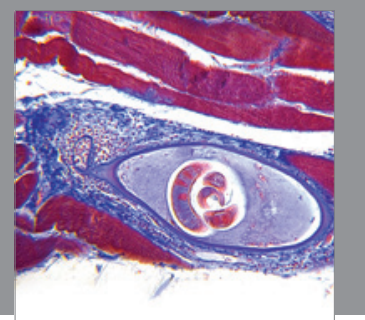

Gastroenterology

Research and Practice
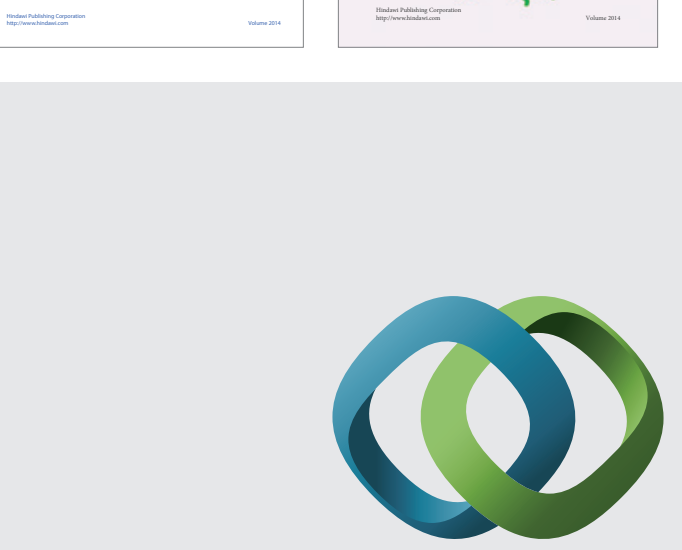

\section{Hindawi}

Submit your manuscripts at

http://www.hindawi.com
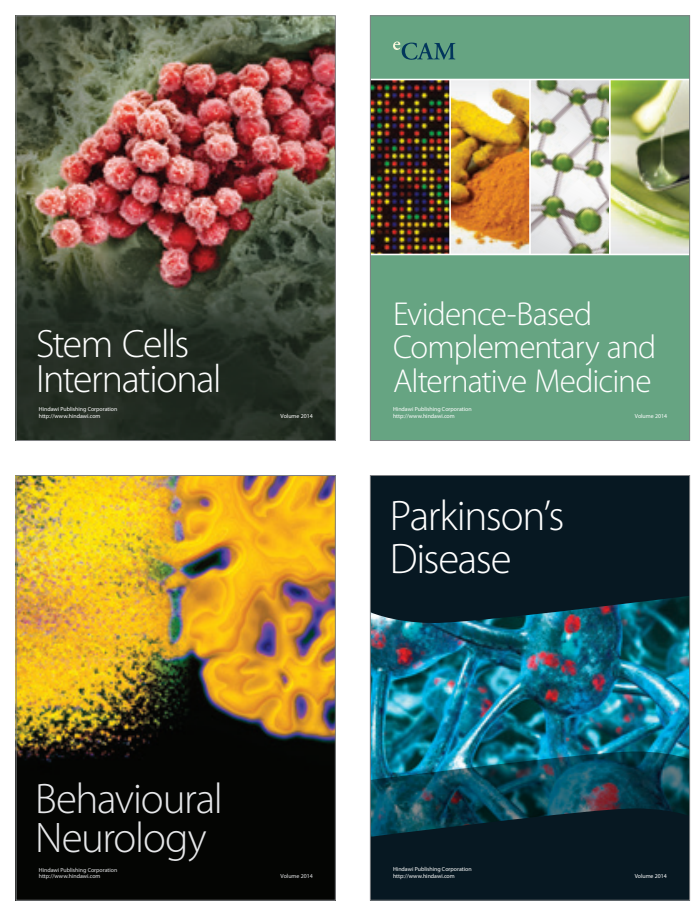

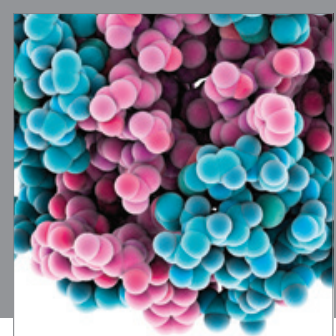

Journal of
Diabetes Research

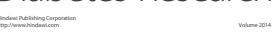

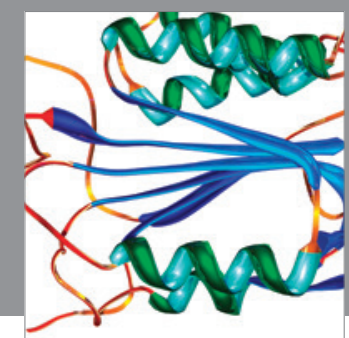

Disease Markers
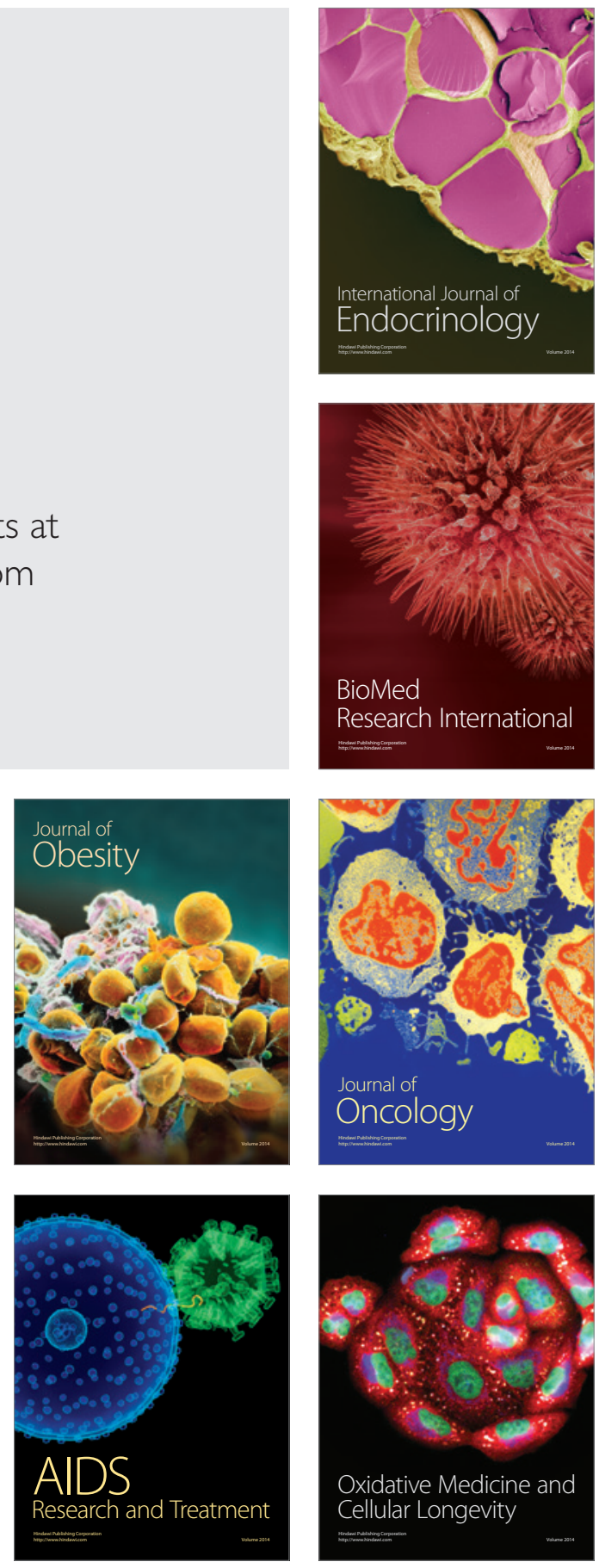